\title{
THE DEVELOPMENT OF A ROBOTIC SYSTEM FOR TOOL DEPLOYMENT IN HAZARDOUS ENVIRONMENTS
}

\author{
Khaled Zied, Derek W. Seward \\ Department of Engineering, Lancaster University, $U K$ \\ k.zied@lancaster.ac.uk, d.seward@lancaster.ac.uk \\ John Riehl and Anthony Dolman \\ Construction Robotics Limited, Trafford Park, Manchester, UK \\ www.constructionrobotics.com
}

\begin{abstract}
The factors that need to be present in order to justify the economic use of robotics in construction are listed. A particular prototype robotic device, Starlifter ${ }^{\circledR}$, is described in some detail. A case study is then presented in which a contract to drill over a thousand holes into the underside of a motorway bridge is analysed. It is concluded that, in this particular case, automated methods would probably be more economic.
\end{abstract}

Keywords: Construction Robotics, Kinematics, Simulation, Sensors, Tool deployment, Economics

\section{INTRODUCTION}

The paper describes a working prototype of a heavy tool manipulator robot, 'Starlifter ${ }^{\circledR}$, which has been specifically designed for use in construction and decommissioning - see figure (1). Consideration is given to the means by which it can be incorporated into the actual work process through computer simulation and the addition of intelligent sensing.

Kangari and Halpine [1] identified the key factors in order to justify the implementation of construction robotics, and recommended that a need-based feasibility study should be carried out. This requires some or all of the following factors to be present:

- A labour intensive process (otherwise it is implied that a reasonable amount of existing conventional mechanisation exists),

- Hazardous to health (this includes the risk of exposure to dust or radiation etc.),

- A physically dangerous process (this includes exposure to moving parts or working sub-sea or at heights.).

For a robotic manipulator such as Starlifter $^{\circledR}$ it is possible to add:

- The need to exploit multiple degrees of freedom (If the task only requires movement about one or two axes then a simpler machine will probably be more economic than a robotic manipulator),

- A substantial mass to manipulate (Construction tasks are rarely repetitive enough to justify the use of a manipulator for light tasks that can be easily accomplished by a human),

- The opportunity to complete the task quicker and reduce disruption to other activities (It is particularly beneficial if the need for support scaffolding and/or road closures is removed),

- Adequate access (work in confined spaces adds significantly to the cost and difficulty of manipulator operations).

It can be argued that unless all or most of the above factors are present, there is little likelihood of a robotic solution being economic.

It is also possible to identify the technologies required to support the implementation of robotics. Esposito et al [2] lists locomotion, automation, task performance, user interface and remote sensors. Griffith et al [3] identifies similar factors in the field of hazardous material handling

The initial application area for Starlifter ${ }^{\circledR}$ has been identified as diamond core drilling and concrete sawing. An example of a specific project where such technology is viable is described later in the form of a case study. A preliminary economic analysis is then carried out to compare the viability of robotic solutions with traditional techniques. 


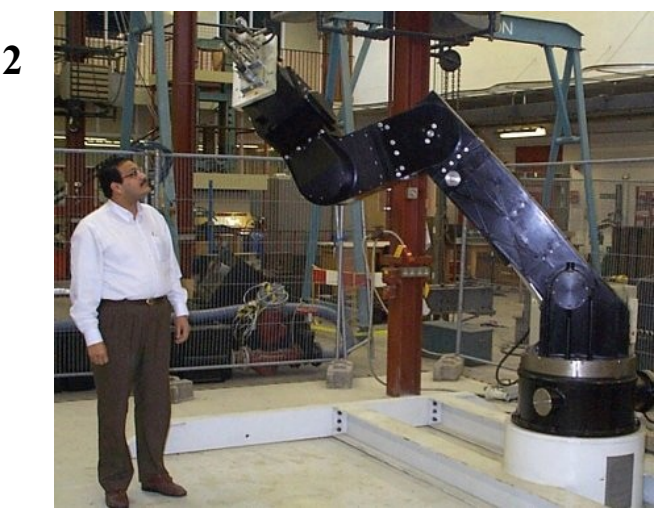

Figure 1

'Starlifter ${ }^{\circledR}$ ' robot

\section{STARLIFTER ${ }^{\circledR}$ DESCRIPTION}

Starlifter is a hydraulically powered portable robot for tool deployment. Figure (1) shows the complete prototype of Starlifter ${ }^{\circledR}$ robotic system. It is a six-degree of freedom manipulator, the kinematic diagram of the arm and the joint configuration are schematically represented in figure (2) and figure (3) respectively. The link parameters are listed in Table (1) according to the well-known Denavit-Hartenburg notation. Other properties include: a- A load carrying capacity of $200 \mathrm{~kg}$ at any orientation of the first joint.

b- The joints can be simultaneously locked in any selected position with power and control shut down to provide a stable platform to deploy heavy duty tooling systems.

c- Fully automatic tool changing capabilities.

d- Fully arterial supplies to toolingmanifold/adapter:

- 200 bar hydraulics

- 3-phase power

- 2-video channels

- 10 tool-function controls

e- Teleoperation control with programmable capability. In conjunction with the on board video camera and automatic tool changing, the machine may be controlled while the operator is distant from the work environment.

Table 1. D-H parameters for Starlifter ${ }^{\circledR}$

\begin{tabular}{cccccc}
\hline link & $\theta_{i}$ & $\alpha_{i}$ & $a_{i}$ & $d_{i}$ & Range \\
\hline 1 & 0 & 90 & 50 & 380 & $-135 \rightarrow+135$ \\
2 & 0 & 0 & 1080 & 0 & $-35 \rightarrow+75$ \\
3 & 0 & 0 & 595 & 0 & $-100 \rightarrow+100$ \\
4 & 0 & -90 & 342 & 0 & $-90 \rightarrow+90$ \\
5 & -90 & -90 & 0 & 0 & $-90 \rightarrow+90$ \\
6 & 0 & 0 & 0 & 471 & $-185 \rightarrow+185$ \\
\hline
\end{tabular}

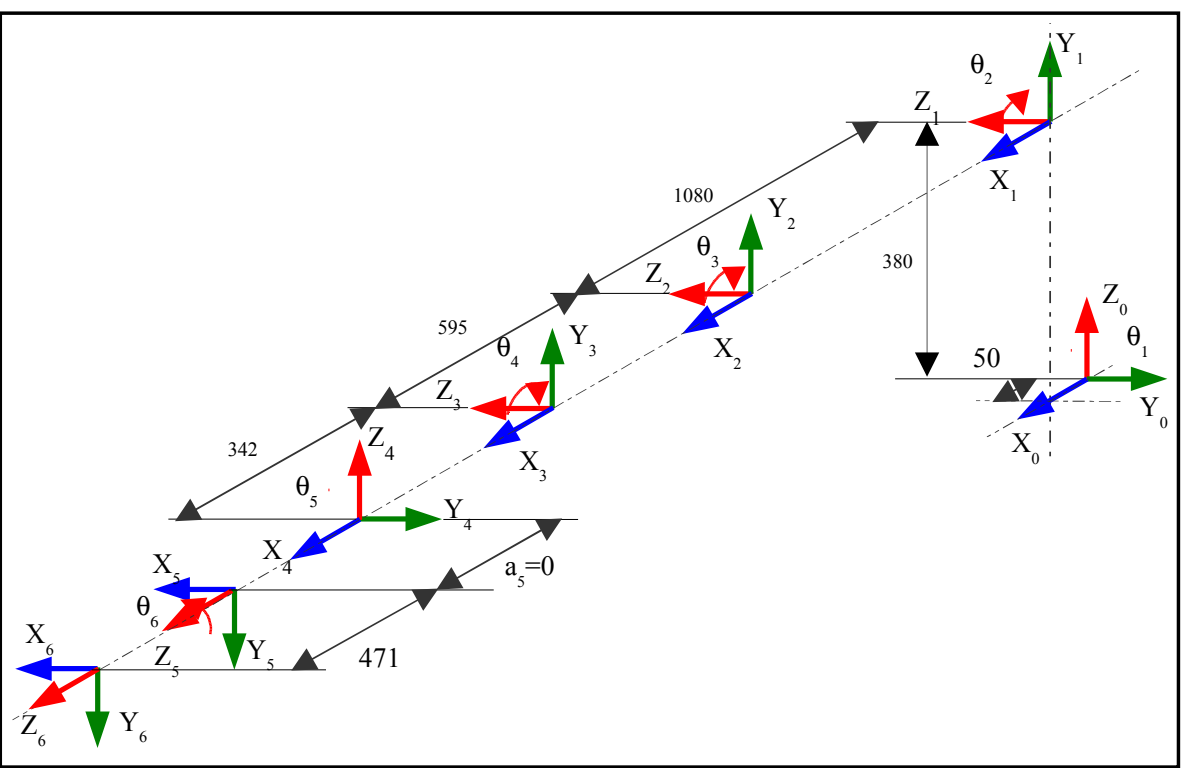

Figure 2. Kinematics of 'Starlifter" ${ }^{\circledR}$, robot 


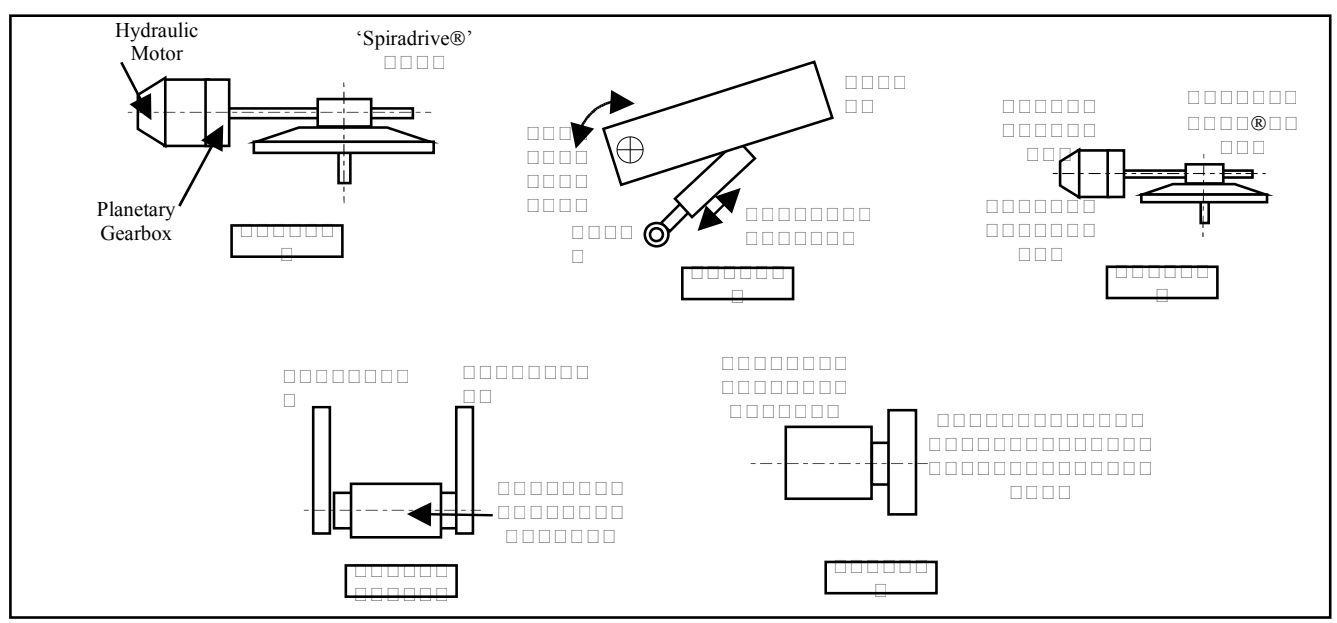

Figure 3. Starlifter ${ }^{\circledR}$ joint configuration

Starlifter $^{\circledR}$ currently utilises the ATC (Advanced Teleoperation Controller) from UK Robotics. This offers enhanced operator control and feedback through the Windows user interface. ATC is supplied as a dedicated and compact unit and uses industrial PC/VME technology to provide a rugged and cost effective system. It features the following: [4]

1. Joint space teleoperation with continuous wrist rotation available.

2. User defined camera frames and tool description

3. Path record, playback and storage.

4. Graphical user interface as shown in figure (4)

5. Twin joystick manipulator control

6. Cartesian control in world and tool coordinates.

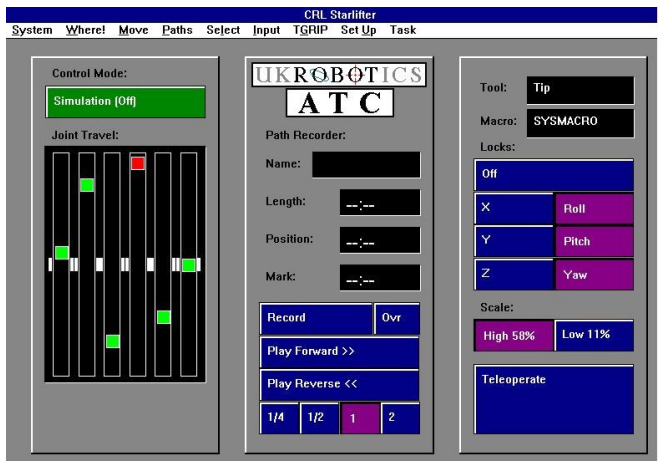

Figure 4. ATC Controller GUI

\section{STARLIFTER ${ }^{\circledR}$ SIMULATION}

A kinematic model of the manipulator has been developed in the robot simulation package 'Workspace' -see figure 5. Such a simulation will be an integral part of the actual work process as discussed in the typical scenario below.

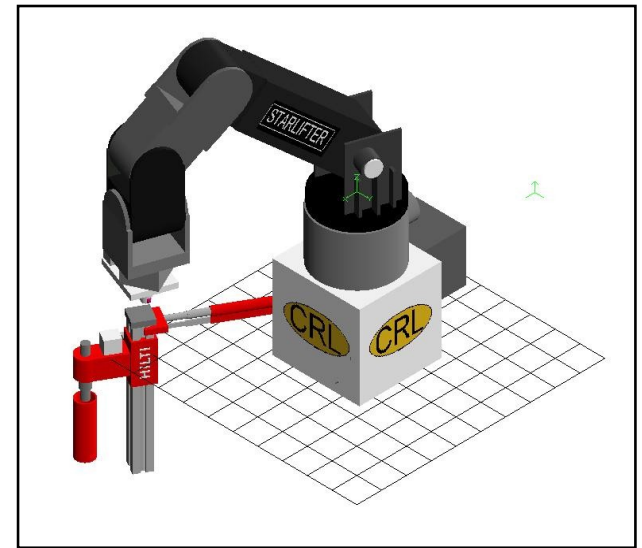

Figure 5. Workspace Simulation

The simulation has been used to investigate critical aspects of the working envelope, such as the size of the plane area that can be scanned by the end effector from a given location of joint 1 .

Workspace has been extended by the addition of a real-time teleoperation module that will enable the real robot to be driven and observed from the PC.

\subsection{Working Envelope of Starlifter ${ }^{\circledR}$}

Figure (6) shows elevation and plan views of the working envelope of Starlifter ${ }^{\circledR}$ without the tool. This envelope will be extended by a distance equal to the tool length in all directions. In case of heavy tools a restriction will be added on the movement of the end effector to avoid collision with the arm. 

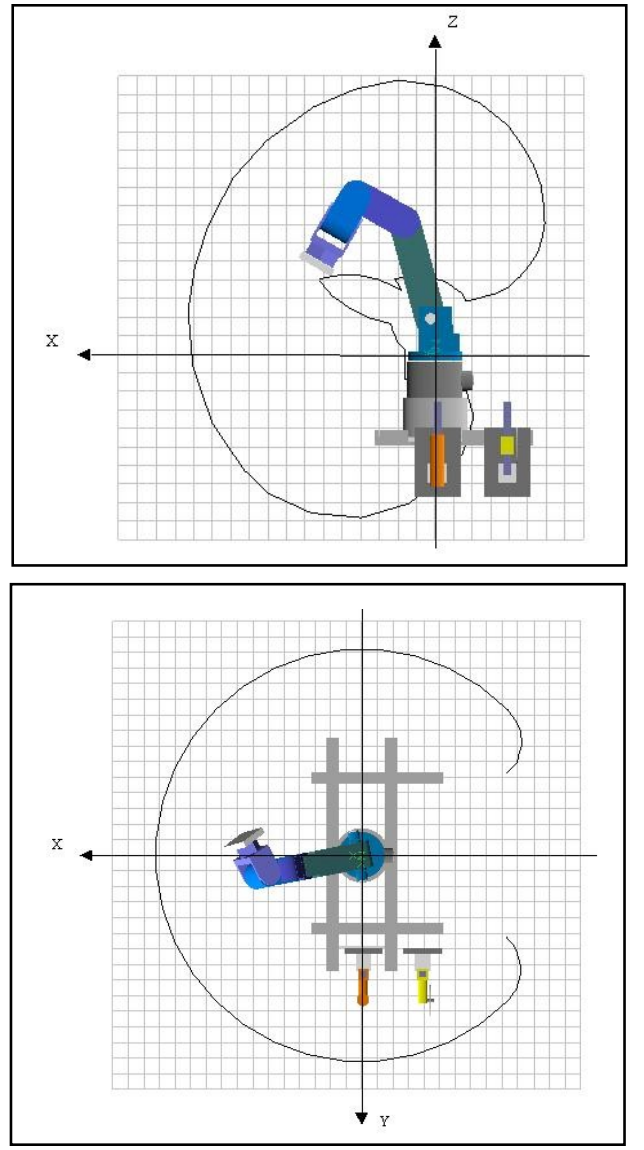

Figure 6. The Working Envelope of Starlifter ${ }^{\circledR}$

\section{CASE STUDY}

\subsection{Methodology}

The industrial partner in this research project, Construction Robotics Limited, is part of a group that includes a major concrete diamond drilling and sawing contractor, Kingstar Contracts Limited. This means that the research has access to a complete archive of financial and performance data from past and current contracts. It is the intention to identify a small number of key projects that meet all or most of the criteria listed in section 1 , and then to carry out a careful analysis of those projects to see which of them could justify the adoption of a robotic tool deployment system such as Starlifter ${ }^{\circledR}$. One partial analysis is reported below.

\subsection{Job Description}

The case study is based around a contract that was completed, using traditional methods, on the M1 motorway in Southern England in 1998.

The main task consists of the drilling of holes in the underside of a major motorway bridge for the purpose of inserting cathodic protection rods, in order to reduce corrosion of the reinforcement bars. The task must avoid drilling through existing reinforcement bars. Key features are:

1. Over 1000 holes required ranging from 300 to $400 \mathrm{~mm}$ in depth

2. Approximate position of holes previously marked on a grid at $1 \mathrm{~m}$ spacing.

3. Original contract drawings too unreliable for accurate location of reinforcement

4. Use of electro-magnetic re-bar locators too time consuming and unreliable.

It can be seen that most of the criteria listed in section 1 have been met.

\subsection{Traditional Approach}

The traditional approach that was actually adopted firstly involved complete scaffolding of the underside of the bridge. After marking-out the approximate hole positions, the drilling task is started by a worker making investigative small diameter holes with a hand drill to check for the presence of reinforcement bars. If any bars are encountered he must move to another location until a reinforcement-free region is found. The main holes are then formed using a core drill-rig. All investigative holes must be made good.

\subsection{Proposed robotic scenario}

Two types of activity are required:

1. Off-line preparation.

2. Site-based operations.

The first of these is office based and will consist of using the simulator to produce off-line programs for the specific tasks. For complex situations this will require a 3-D model of the operating environment to be modelled. This will enable robot movements to be pre-programmed in order to facilitate collision avoidance. The 3-D models may be obtained from contract drawings, photographs or by utilising some form of laser scanning system. This stage can also provide valuable visual presentations of the work to both clients and operators, as well as vital information for work timing, scheduling and costing.

The proposed scenario for site-based operations is considered in relation to the previously mentioned support technologies identified by Esposito [2]

\section{Locomotion}

Because, unlike conventional industrial robots, Starlifter ${ }^{\circledR}$ can operate in any orientation it is proposed that it be connected to a conventional hydraulic telescopic crane as shown in figure (7). The robot is transported to site on an independent truck and positioned such that the telescopic crane boom can reach its base adapter. 


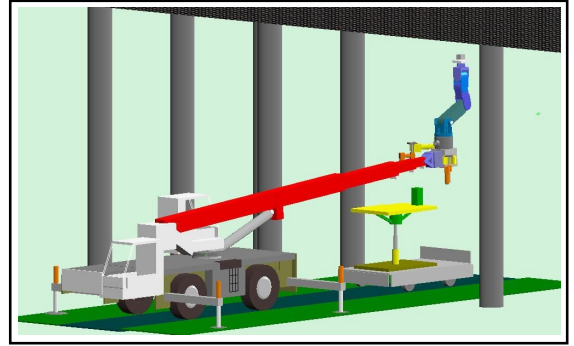

Figure 7. Telescopic crane mounting

The telescopic boom picks up the robot and is controlled by a ground based operator until the end effector is, say, within a metre of the target hole position.

\section{Remote sensors}

At this stage the end effector consists of a sensing head. This includes:

- a TV camera to give a clear image of the target area,

- one or more ultrasonic sensors for precise distance measurement. Thus the position and orientation of the end effector relative to the work surface can be determined. These sensors may also be useful for collision avoidance.

- a reinforcement bar locator such as the Hilti Feroscan.

Work is currently underway to develop a sensing head using a set of range sensors, re-bar locator and a TV camera. A preliminary design is shown in figure (8).

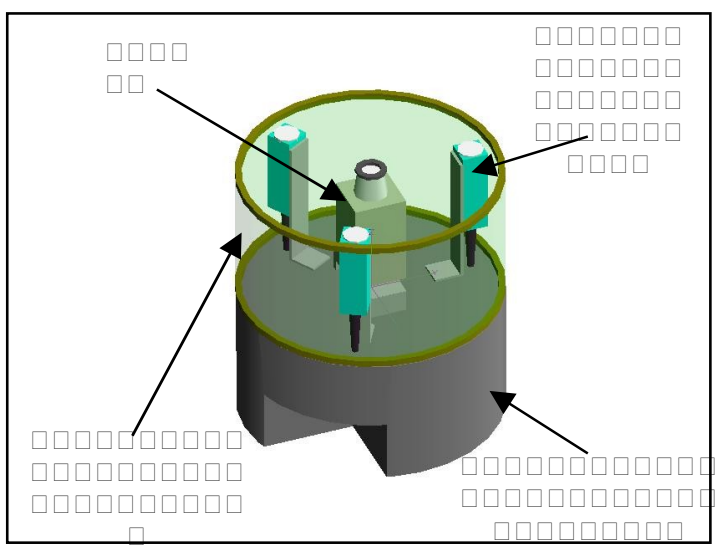

Figure 8 Preliminary sensing head design

\section{Automation}

The operator will identify the required hole target and the sensing head will be automatically adjusted to move to a point which is a fixed distance from the target and to be parallel to the work surface. The reinforcement bar locator will then be used to identify a clear zone close to the target. Once the accurate hole position has been identified and memorised, the automatic tool changer is used to switch to the drilling head.

\section{Task performance}

The operator will have at their disposal a library of application routines to handle commonly occurring tasks. Hence in this case, only the depth of the hole will need to be input to the controller. A further video camera will enable the operator to monitor progress from the ground. A record of all robot movements will be logged in order to provide complete traceability of the operation.

\section{User interface}

The user interface will be task specific and provide video monitoring of the robot together with the possibility of the Workspace simulator providing a virtual model of the robot which will mimic the actual robot in real time. It is important that the user interface permits the subtle combination of remote control, tele-robotic control and automatic control.

Figure (9) illustrates a typical system architecture in the form suitable for research. A final commercial system will contain only one PC monitor with each task being contained within a bespoke ATC "application".

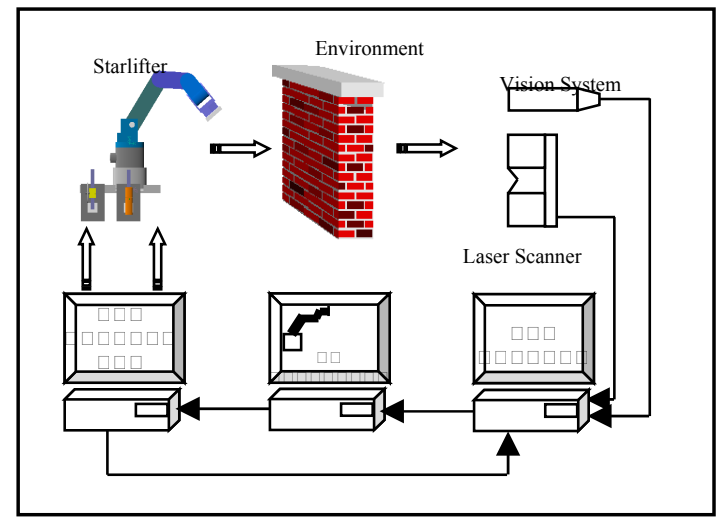

Figure 9

System architecture for automated process

\subsection{Economic Analysis}

An approximate estimation of the costs involved in the traditional operation and the automated operation is carried out for the purpose of comparison.

\section{Traditional operation}

The following data is extracted from the actual operational files: 


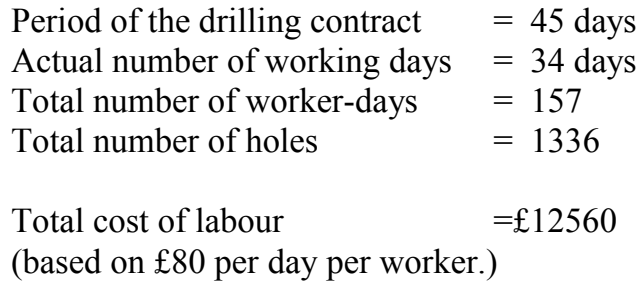

Cost for transportation and tool kits $=£ 29045$ (based on a hiring cost of $£ 185$ per day for all equipment and transportation van per worker)

Total cost $=12560+29045$

$=£ 41605$

(The above excludes overhead costs)

Also to the above must be added the cost of scaffolding for the whole site.

\section{Robotic operation}

Average number of drilled holes/day $=50$

(conservative approach)

Total period for drilling 1336 hole $=27$ days

Number of operators per day $\quad=2$

Estimated daily cost of hiring the robot $=£ 600$

(includes equipment and based on the estimation of capital cost and maintenance.)

Total cost of robot $=£ 16200$

Cost of hiring positioning vehicle/day $=£ 400$

Total cost of hiring positioning vehicle $=£ 10800$

Total cost of labour $\quad=£ 8640$

Total cost $8640+10800+16200$ $=\mathfrak{\$ 3 5 6 4 0}$

\section{Results}

Time saved $=34-27=7$ days

Cost difference $=41605-35640=£ 5965$

(in favour of the automated approach)

\section{CONCLUSIONS}

It can be concluded from the above that the robotics approach would probably have been cost effective. In the case of this contract it can be seen that it:

- did not require the motorway to be closed and hence was not time critical,

- was not particularly hazardous,

- was possible for a human to physically handle the drilling equipment,

- could have been achieved by a simpler device with less degrees of freedom if operated from a hydraulic access platform.

If any of those constraints had in fact prevailed, the case for a robotics method would have been even stronger.

It is clear that robotic devices are essential in highly hazardous environments, however a more careful analysis must be carried out for further case studies to identify the critical factors that must be present in order that robotic devices such as Starlifter $^{\circledR}$ represent a clearly economic solution in general construction.

The next type of contract to be investigated will be concrete sawing which uses heavier equipment that cannot be hand-held and often requires more degrees of freedom to achieve the necessary cut shape. It is anticipated that such applications will indicate an even more favourable outcome for robotics.

\section{REFERENCES}

1. Kangari, R. and Halpin, D. W., 1990, "Identification of factors influencing implementation of construction robotics.", Journal of Construction Management and Economics, V8, pp 89-104.

2. Esposito, C. Sullivan D., Frank U. and Cibulskis R., " Field application of robotic systems in hazardous waste site operation.", Robotics and remote system for hazardous environments, Jamshidi, M. and Eicker, P. [Eds], PTR Prentice Hall, Englewood Cliffs, New Jersey, 1-31.

3. Griffith, A., Haas, C., and Tucker, R., "Feasibility Analysis of Automated Material Handling for Hazardous Waste Remediation," Proceedings of the ASCE Conference on Robotics for Challenging Environments, Albuquerque, NM, pp. 338346, March 1994.

4. UK Robotics Advanced Teleoperation controller for the Starlifter ${ }^{\circledR}$ Manipulator, User Manual 1998. UKRL.95.024 Issue 1 